\title{
The Chemo-Dynamical History of the Milky Way as Revealed by SDSS/SEGUE
}

\author{
Timothy C. Beers ${ }^{1,2}$ \\ ${ }^{1}$ Department of Physics \& Astronomy, Michigan State University, \\ email: beers@pa.msu.edu \\ ${ }^{2}$ Joint Institute for Nuclear Astrophysics
}

\begin{abstract}
Although originally conceived as primarily an extragalactic survey, the Sloan Digital Sky Survey (SDSS-I), and its extensions SDSS-II and SDSS-III, continue to have a major impact on our understanding of the formation and evolution of our host galaxy, the Milky Way. The sub-survey SEGUE: Sloan Extension for Galactic Exploration and Understanding, excuted as part of SDSS-II, obtained some 3500 square degrees of additional ugriz imaging, mostly at lower Galactic latitudes, in order to better sample the disk systems of the Galaxy. Most importantly, it obtained over 240,000 medium-resolution spectra for stars selected to sample Galactocentric distances from 0.5 to $100 \mathrm{kpc}$. In combination with stellar targets from SDSS-I, and the recently completed SEGUE-2 program, executed as part of SDSS-III, the total sample of SDSS spectroscopy for Galactic stars comprises some 500,000 objects.

The development of the SEGUE Stellar Parameter Pipeline has enabled the determination of accurate atmospheric parameter estimates for a large fraction of these stars. Many of the stars in this data set within $5 \mathrm{kpc}$ of the Sun have sufficiently well-measured proper motions to determine their full space motions, permitting examination of the nature of much more distant populations represented by members that are presently passing through the solar neighborhood. Ongoing analyses of these data are being used to draw a much clearer picture of the nature of our galaxy, and to supply targets for detailed high-resolution spectrscopic follow-up with the worlds largest telescopes. Here we discuss a few highlights of recently completed and ongoing investigations with these data.
\end{abstract}

Keywords. astronomical data bases: surveys, Galaxy: halo, structure, methods: data analysis, stars: abundances

\section{Introduction}

The era of the massive surveys of Galactic stars is now very much underway. Our understanding of the history of the formation and evolution of the stellar populations in the Galaxy is presently being revolutionized, based on results from two primary surveys, the Sloan Digital Sky Survey (SDSS; York et al. 2000), in particular its dedicated sub-surveys Sloan Extension for Galactic Exploration and Understanding (SEGUE-1 and SEGUE-2; Yanny et al. 2009) and the RAdial Velocity Experiment (RAVE; Zwitter et al. 2008; Wyse, this volume). Collectively, these two surveys are in the process of providing detailed spectroscopic information for a million or more stars, sampling all of the known stellar populations in the Galaxy. Although refinements in the procedures for extracting estimates of the stellar atmospheric parameters $\left(\mathrm{T}_{\text {eff }}, \log \mathrm{g},[\mathrm{Fe} / \mathrm{H}]\right)$ and individual element abundances (in the case of SDSS/SEGUE, $[\alpha / \mathrm{Fe}]$ and $[\mathrm{C} / \mathrm{Fe}]$ ratios; for RAVE, many more) are still underway, the wealth of information already available provides the 


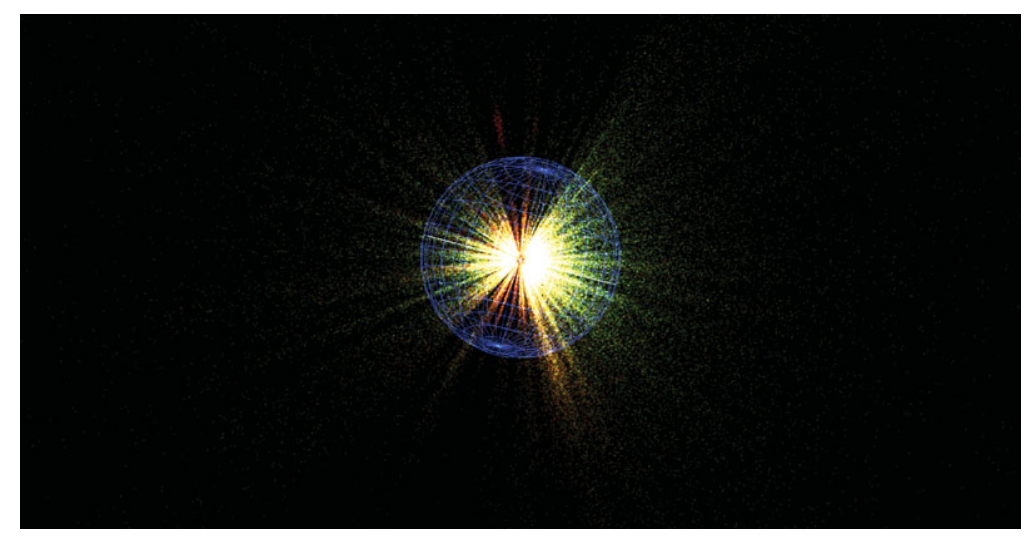

Figure 1. Simple visualization of the SDSS/SEGUE stellar database, for roughly 400,000 stars with available atmospheric parameter estimates from the SSPP. The sphere has a radius of 10 $\mathrm{kpc}$, centered on the Sun. The colors scale with metallicity, from green (metal-poor stars) to red (metal-rich stars).

basis for numerous investigations and follow-up observations. $\dagger$ Here we summarize a few of the ongoing projects that are being enabled by the SDSS/SEGUE database.

\section{The SEGUE Stellar Parameter Pipeline and a Visualization of the SDSS/SEGUE Stellar Database}

The SEGUE Stellar Parameter Pipeline (SSPP) processes the wavelength- and fluxcalibrated spectra generated by the standard SDSS spectroscopic reduction pipeline, obtains equivalent widths and/or line indices for about 80 atomic or molecular absorption lines, and estimates the effective temperature, $\mathrm{T}_{\text {eff }}$, surface gravity, log g, and metallicity, $[\mathrm{Fe} / \mathrm{H}]$, for a stellar spectrum through the application of a number of approaches. A given method is usually optimal over specific ranges of color and signal-to-noise $(S / N)$ ratio. The SSPP employs 8 primary methods for the estimation of $\mathrm{T}_{\text {eff }}, 10$ for the estimation of $\log \mathrm{g}$, and 12 for the estimation of $[\mathrm{Fe} / \mathrm{H}]$. The final estimates of the atmospheric parameters are obtained by robust averages of the methods that are expected to perform well for the color and $S / N$ of the spectrum obtained for each star. The use of multiple methods allows for empirical determinations of the internal errors for each parameter, based on the range of reported values from each method - typical internal errors for stars in the temperature range that applies to the calibration stars are $\sigma_{\text {Teff }} \sim 100 \mathrm{~K}$ to $\sim 125$ $\mathrm{K}, \sigma_{\log g} \sim 0.25$ dex, and $\sigma_{[\mathrm{Fe} / \mathrm{H}]} \sim 0.20$ dex. The external errors in these determinations are of similar size. See Lee et al. (2008a), Lee et al. (2008b), and Allende Prieto et al. (2008) for more details.

Over the past several years, large-aperture telescopes have been used to obtain highresolution spectroscopy for over 300 of the brighter $\left(14.0<g_{0}<17.0\right)$ SDSS stars (see Allende Prieto et al. 2008; Aoki et al., in preparation; Lai et al., in preparation). The observations reported by Aoki et al. and Lai et al. suggest that the current SSPP is actually somewhat conservative in the assignment of metallicities for stars of the lowest $[\mathrm{Fe} / \mathrm{H}]$, in the sense that high-resolution estimates of $[\mathrm{Fe} / \mathrm{H}]$ are on the order of $0.3 \mathrm{dex}$ lower than those reported by the SSPP.

$\dagger$ The most recent public release for SDSS/SEGUE is DR-7, which includes SEGUE-1; see Abazajian et al. (2009). The next public release for SDSS/SEGUE is DR-8, scheduled for December 2010, which will include results from SEGUE-2. 
Based on the derived atmospheric parameters from the SSPP, and distances estimated from photometric parallaxes, Figure 1 shows a simple visualization of the SDSS/SEGUE database. The colors are coded to represent metallicity, and clearly indicate the presence of stars from the disk populations close to the Galactic plane, and an extended population of stars comprising representatives of the inner- and outer-halo populations. More sophisticated visualizations, encoding other available observables such as full space motions, are presently being developed.

\section{The Impact of SDSS/SEGUE on Searches for Metal-Poor Stars}

The great majority of metal-poor stars in the Galaxy identified to date have come from two primary sources, the HK survey of Beers and colleagues (Beers et al. 1985; Beers et al. 1992) and the Hamburg/ESO Survey of Christlieb and colleages (Christlieb et al. 2008). SDSS/SEGUE specifically targeted large numbers of low-metallicity candidates based on combinations of SDSS filters that approximately isolate them from the vastly more common higher metallicity stars of the disk populations. This approach, although not as selective as prism-survey techniques, has greatly enlarged the numbers of known metal-poor stars, as summarized in Table 1. It should be kept in mind that, due to the ongoing calibration of the SSPP at lower metallicities, the true numbers of extremely metal-poor stars identified by SDSS/SEGUE with $[\mathrm{Fe} / \mathrm{H}]<-3.0$ is certain to increase in the near future. The apparent lack of newly discovered ultra $([\mathrm{Fe} / \mathrm{H}]<-4.0)$ and hyper $([\mathrm{Fe} / \mathrm{H}]<-5.0)$ metal-poor stars by SDSS/SEGUE could also be, at least in part, due to calibration issues. In order to be certain, all stars with metallicities suggested by the SSPP to be below $[\mathrm{Fe} / \mathrm{H}]=-3.0$ need to be observed at higher spectral resolution, as the presence of interstellar Ca II K (the only metallic feature detectible at such low metallicities from medium-resolution spectra) can confound the derived abundance as well.

Table 1. Numbers of known metal-poor stars pre- and post-SDSS/SEGUE

\begin{tabular}{lcrr}
\hline Metallicity Class & $<[\mathbf{F e} / \mathbf{H}]$ & $\mathbf{N}$ (Pre-SDSS) & N (Post-SDSS) \\
\hline Metal-Poor & $<-1.0$ & 15,000 & $150,000+$ \\
Very Metal-Poor & $<-2.0$ & 3,000 & $30,000+$ \\
Extremely Metal-Poor & $<-3.0$ & 400 & $1,000+$ \\
Ultra Metal-Poor & $<-4.0$ & 5 & 5 \\
Hyper Metal-Poor & $<-5.0$ & 2 & 2 \\
Mega Metal-Poor & $<-6.0$ & 0 & 0 \\
\hline
\end{tabular}

\section{An Update on the Inner/Outer-Halo Dichotomy}

Based on an analysis of a large sample of calibration stars from SDSS DR-5 (AdelmanMcCarthy et al. 2007), Carollo et al. (2007) argued for the existence of at least a twocomponent halo. In their view, the Galactic halo comprises two broadly overlapping structural components, an inner and an outer halo. These components exhibit different spatial density profiles, stellar orbits, and stellar metallicities. It was found that the inner-halo component dominates the population of halo stars found at distances up to $10-15 \mathrm{kpc}$ from the Galactic center, while the outer-halo component dominates in the region beyond $15-20 \mathrm{kpc}$. The inner halo was shown to comprise a population of stars exhibiting a flattened spatial density distribution, with an inferred axial ratio on the order of 0.6. According to these authors, inner-halo stars possess generally high orbital eccentricities, and exhibit a small (or zero) net prograde rotation around the center of the 

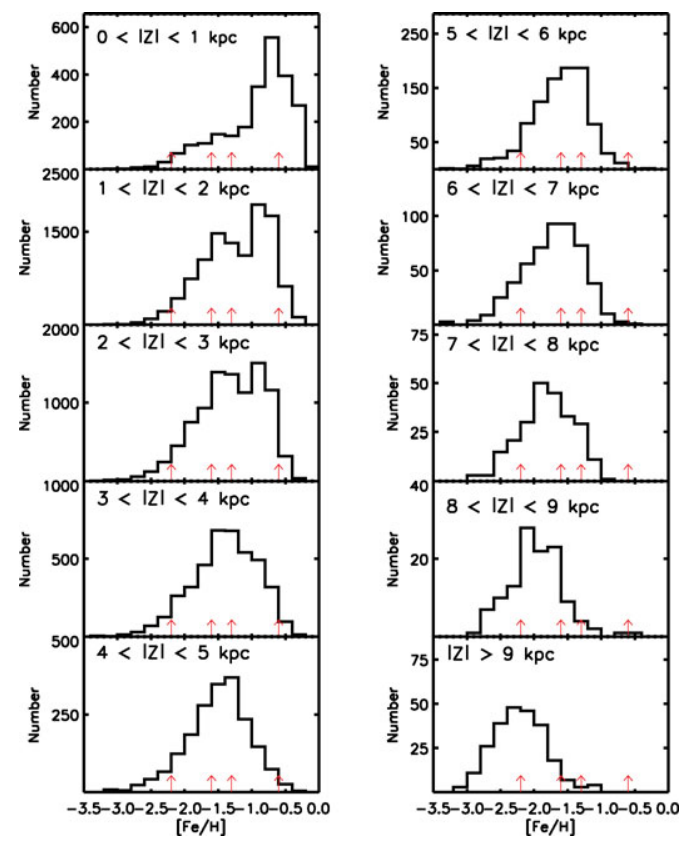

Figure 2. Observed metallicity distribution functions (MDFs) for the full sample of SDSS/SEGUE DR-7 calibration stars as a function of vertical distance from the Galactic plane. The black histograms represent the MDFs obtained at different cuts of $|Z|$, while the red arrows denote the locations of the metallicity peaks of the MDF for the thick disk $(-0.6)$, the MWTD $(\sim-1.3)$, the inner halo $(-1.6)$, and the outer halo $(-2.2)$, respectively.

Galaxy. The metallicity distribution function (MDF) of the inner halo peaks at $[\mathrm{Fe} / \mathrm{H}]$ $=-1.6$, with tails exending to higher and lower metallicities. By comparison, the outer halo comprises stars that exhibit a more spherical spatial density distribution with an axial ratio $\sim 0.9$. Outer-halo stars possess a wide range of eccentricities, exhibit a clear retrograde net rotation, and are drawn from an $\mathrm{MDF}$ that peaks at $[\mathrm{Fe} / \mathrm{H}]=-2.2$, a factor of four lower than that of the inner-halo population.

This work has now been extended by Carollo et al. (2009) (see also Carollo's article, this volume), making use of the calibration star sample through DR-7. The full sample of 32360 stars represents an increase of $60 \%$ relative to the numbers used in the previous analysis. The larger sample, which includes many more stars from the disk populations due to SEGUE observations, has been used to derive, among many other results, estimates of the velocity ellipsoids for the inner- and outer-halo populations, as well as for the metal-weak thick disk (MWTD) and canonical thick-disk populations.

One can obtain an impression of the contribution of the various populations to the observed MDF from inspection of Figure 2, which shows its variation with height above the Galactic plane. Examination of the left-hand column of panels in this figure shows how the MDF changes from the upper panel, in which there are obvious contributions from the thick-disk, MWTD, and inner-halo components in the cuts close to plane, to the lower panel, with an MDF dominated by inner-halo stars. In the right-hand column of panels, with distances from the plane greater than $5 \mathrm{kpc}$, the transition from innerhalo dominance to a much greater contribution from outer-halo stars is obvious. This demonstration is, by design, independent of any errors that might arise from derivation of the kinematic parameters, and provides confirmation of the difference in the chemical 
properties of the inner- and outer-halo populations originally suggested by Carollo et al. (2007).

Although it might appear possible to attempt mixture-model analysis to obtain MDFs for each of the individual components, we recall that biases in the selection of the stars in the calibration-star sample would confound such an attempt. Other samples of SDSS stars are being studied for this purpose, and will be reported on in due course.

\section{ECHOS in the Inner Halo}

Schlaufman et al. (2009) have used the SEGUE spectroscopic database of metal-poor main-sequence turnoff (MPMSTO) stars to search for significant radial velocity enhancements along 137 SEGUE-1 lines of sight. They identify ten (seven for the first time) Elements of Cold Halo Substructure (ECHOS) in the volume within $17.5 \mathrm{kpc}$ of the Sun, in the inner halo of the Milky Way. These ECHOS represent the observable stellar debris of ancient merger events in the stellar accretion history of the Milky Way.

These authors use their detections and completeness estimates to infer a formal upper limit of $0.34 \pm 0.2$ on the fraction of the MPMSTO population in the inner halo that belong to ECHOS. They also suggest that there exists a significant population of low fractional overdensity ECHOS in the inner halo, and predict that one-third of the inner halo (by volume) harbors ECHOS with MPMSTO number densities $n \approx 15 \mathrm{kpc}^{-3}$. In addition, they estimate that there are $\approx 10^{3}$ ECHOS in the entire inner halo. Since ECHOS are likely older than the known substructures identified by surface-brightness contrast methods, these detections provide a direct measure of the accretion history of the Milky Way in a region and time interval that has yet to be fully explored. The authors argue, on the basis of this information, that the level of merger activity has been roughly constant over the past few Gyrs, and that there has been no accretion of stellar systems more massive than a few percent of the Milky Way mass in that interval.

\section{6. $[\alpha / \mathbf{F e}]$ Ratios for SDSS/SEGUE Stars}

Lee et al. (in preparation) demonstrate, by comparison with measured abundances of $[\alpha / \mathrm{Fe}]$ from high-resolution spectra in the ELODIE spectral library and recently obtained $R=15000$ spectra from Hobby-Eberly Telescope observations of SDSS/SEGUE stars, that it is possible to determine $[\alpha / \mathrm{Fe}]$ ratios from SDSS spectra (with $S / N>20$ ) to an accuracy of better than $0.1 \mathrm{dex}$, for stars with atmospheric parameters in the range $\mathrm{T}_{\text {eff }}$ $=[4500,7500] \mathrm{K}, \log \mathrm{g}=[1.5,5.0]$, and $[\mathrm{Fe} / \mathrm{H}]=[-2.5,+0.2]$. This capability opens up the opportunity to make use of this ratio to investigate predictions from simulation studies of the formation and evolution of the disk systems of the Milky Way (e.g., Abadi et al. 2003; Brook et al. 2007; Kazantzidis et al. 2008; Roškar et al. 2008; Schönrich \& Binney 2009a; Schönrich \& Binney 2009b), as well as of the halos of the Galaxy (e.g., Johnston et al. 2008).

For example, the lower right panel of Figure 3 shows, for a sample of some 10000 SDSS/SEGUE F- and G-dwarfs in the solar neighboorhood, that stars with higher $[\alpha / \mathrm{Fe}]$ ratios (associated with the thick disk) have orbital eccentricities that peak around 0.2 , with relatively few such stars having higher eccentricities (most of these are found at lower $[\mathrm{Fe} / \mathrm{H}]$, indicating possible membership in the MWTD population). The association of the low-metallicity, high- $[\alpha / \mathrm{Fe}]$ stars with the MWTD population is also indicated by the lower left panel of Figure 3, where it is clear that they exhibit a substantially lower mean rotational velocity. Much remains to be explored with data such as these. 


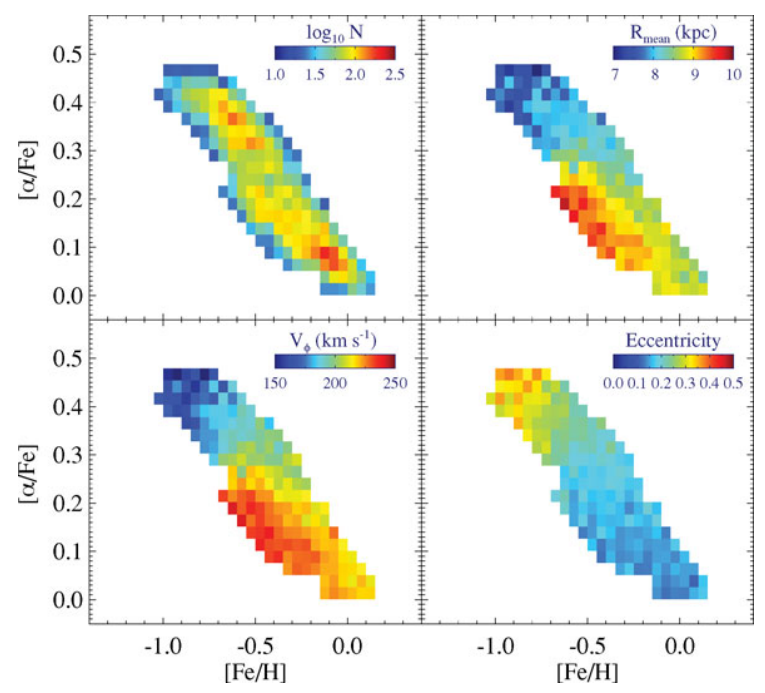

Figure 3. Distribution of number densities, mean orbital radii, rotation velocities, and orbital eccentricities for F- and G-type dwarfs within $2 \mathrm{kpc}$ of the Galactic plane, located between $8<\mathrm{R}<9 \mathrm{kpc}$, in bin sizes of 0.05 dex in $[\mathrm{Fe} / \mathrm{H}]$ by 0.025 dex in $[\alpha / \mathrm{Fe}] . R_{\text {mean }}$ is an average of $R_{\mathrm{max}}$ and $R_{\mathrm{min}}$, the maximum and minimum projected distance on the plane reached by a star over the course of its orbit. Each pixel has more than 20 stars.

\section{7. $[\mathrm{C} / \mathrm{Fe}]$ Ratios for SDSS/SEGUE Stars}

Stars with observed carbon enhancements are likely to play a key role in our understanding of the formation and evolution of the various stellar populations in the Galaxy, as the production mechanisms of carbon depend sensitively on the initial mass function and star formation environments of their birth. Although carbon-enhanced stars were not specifically selected for in SDSS/SEGUE, they are found in great numbers over a variety of the categories that were targeted, such as metal-poor stars, F-turnoff stars, and late-type giants.

Over the course of the past few years, members of the SEGUE team have been working to develop and refine methods for estimation of $[\mathrm{C} / \mathrm{Fe}]$ from the SDSS spectra, with the goal of providing this information for as many stars as possible in the upcoming DR8 public release. For spectra with sufficient $\mathrm{S} / \mathrm{N}$ (roughly $>15 / 1$ ) these methods are now able to estimate $[\mathrm{C} / \mathrm{Fe}]$, or upper limits on $[\mathrm{C} / \mathrm{Fe}]$, accurate to roughly 0.1 dex. See Figure 4 for some examples of fits to the $\mathrm{CH}$ G-band region, which is the feature used to estimate $[\mathrm{C} / \mathrm{Fe}]$. There are several hundred thousand stars in SDSS/SEGUE for which such estimates now exist. Based on these data, Sivarani et al. (in preparation) is obtaining a definitive estimate of the much-debated frequency of Carbon-Enhanced Metal-Poor (CEMP) stars, and in particular, the dependence of this fraction on $[\mathrm{Fe} / \mathrm{H}]$ between solar and $[\mathrm{Fe} / \mathrm{H}]=-3.0$. Carollo et al. (in preparation) is examining whether or not the fraction of CEMP stars differs among the various disk and halo stellar populations identified in the SDSS/SEGUE calibration stars, which has been argued by Tumlinson (2007) to probe the influence of the CMB on the IMF at early times.

\section{Subdwarf $M$ stars in SDSS/SEGUE}

Lepine (2009) and collaborators have been actively pursuing techniques for the identification and analysis of subdwarf M stars based on SDSS colors, and have demonstrated 

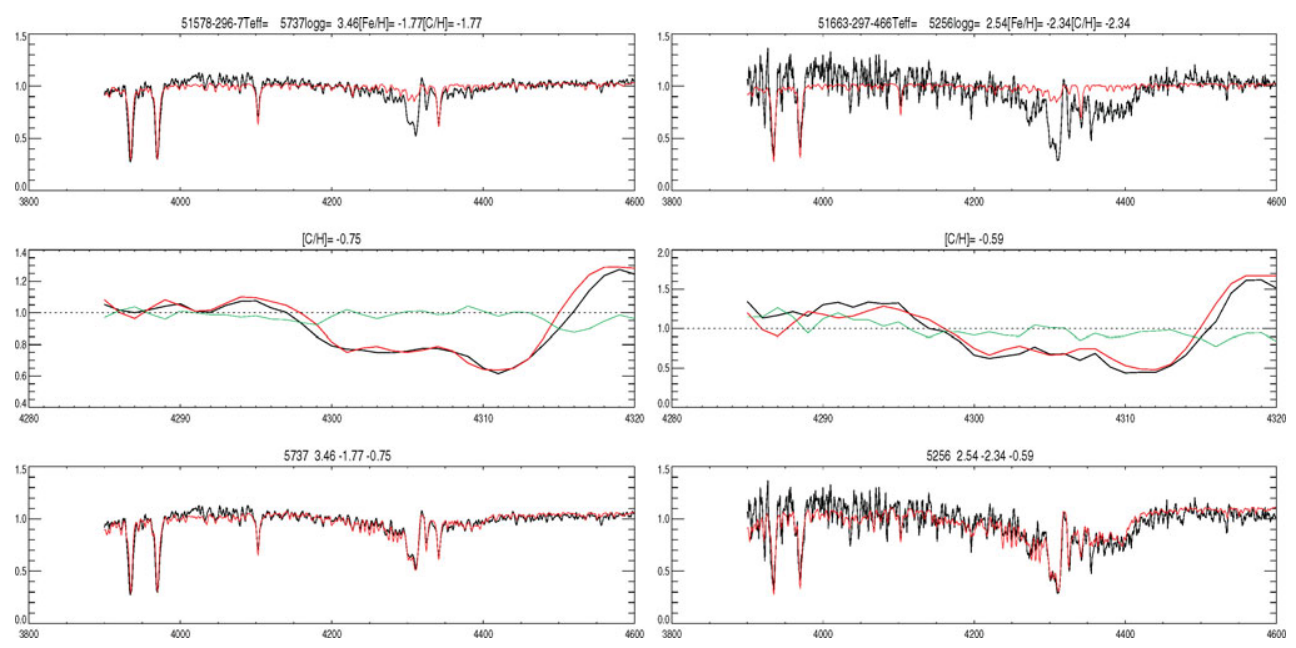

Figure 4. Two examples of the fitting procedure for determination of $[\mathrm{C} / \mathrm{Fe}]$ in metal-poor stars. The upper panels show a portion of the original spectrum (black) overlayed with a synthetic spectrum (red) with the listed parameters and $[\mathrm{C} / \mathrm{Fe}]=0$. The middle panels show the region around the $\mathrm{CH}$ G-band, with a red line showing the best fit. The green line is a division of the original spectrum by the fit spectrum, which should be close to 1.0 for a successful fit. The lower panels show the result of the best fit with the listed $[\mathrm{C} / \mathrm{H}]$ level Left: A moderately metal-poor star. Right: A very metal-poor star.

that they can be usefully separated into metallicity sub-classes, which they refer to as "subdwarfs" (sdM), "extreme subdwarfs" (esdM), and "ultra subdwarfs" (usdM), in order of decreasing metal content. Such stars were specifically targeted in SEGUE-2. As a result of this follow-up, there are now more than 6000 such stars known, an order of magnitude increase in the numbers that were known just a few years ago.

Because sdM, esdM, and usdM stars are selected in a completely different way than other probes in SDSS/SEGUE, they provide a useful comparison to kinematic analyses of, e.g., the calibration stars studied by Carollo et al. (2007) and Carollo et al. (2009). It is thus of great interest to note, as shown in Figure 5, that the kinematics of this very local sample reflects the dichotomy of the halo that has been previously claimed. As can be appreciated from inspection of this figure, the sdM stars (the centroid indicated by the smaller circle) have local kinematics that can be associated with the thick disk, the esdM stars (the centroid indicated by the large circle) with the inner-halo population, and of greatest interest, the tendency of the usdM stars to exhibit a net retrograde rotation, which can be associated with the outer-halo population.

Funding for SDSS-III has been provided by the Alfred P. Sloan Foundation, the Participating Institutions, the National Science Foundation, and the U.S. Department of Energy. The SDSS-III web site is http://www.sdss3.org/.

SDSS-III is managed by the Astrophysical Research Consortium for the Participating Institutions of the SDSS-III Collaboration including the University of Arizona, the Brazilian Participation Group, University of Cambridge, University of Florida, the French Participation Group, the German Participation Group, the Michigan State/Notre Dame/ JINA Participation Group, Johns Hopkins University, Lawrence Berkeley National Laboratory, Max Planck Institute for Astrophysics, New Mexico State University, New York University, the Ohio State University, University of Portsmouth, Princeton University, University of Tokyo, the University of Utah, Vanderbilt University, University of Virginia, University of Washington and Yale University. 

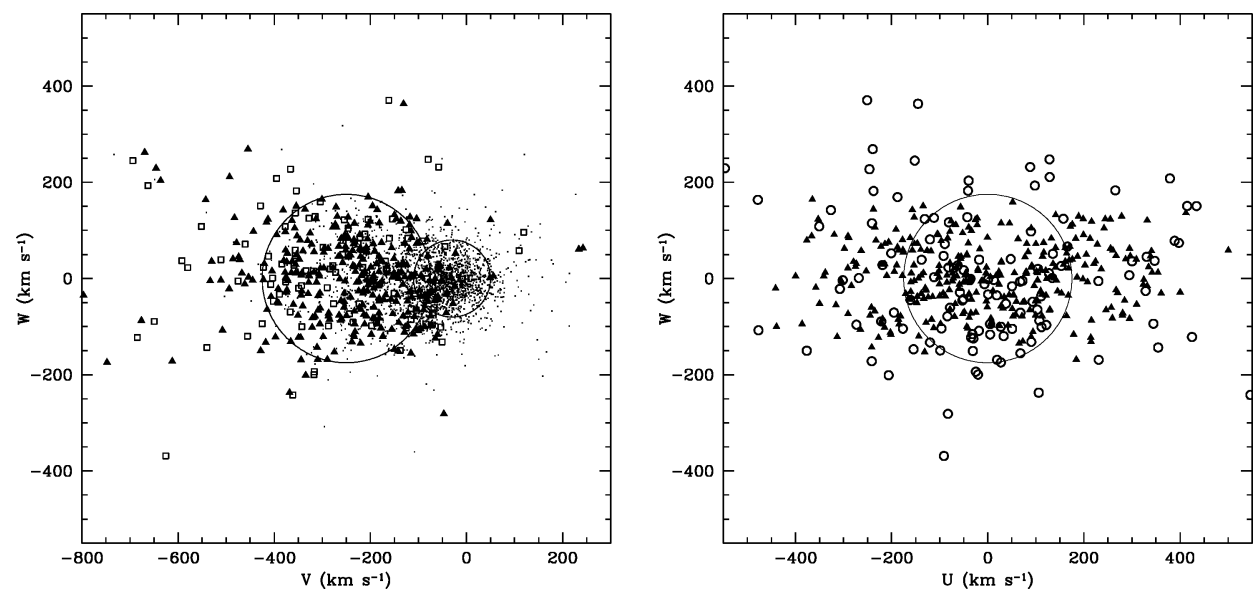

Figure 5. Left: Distribution of $W$ vs. $V$ velocities for the sample of sdM stars (small dots), esdM stars (filled triangles), and usdM stars (open squares) from SDSS/SEGUE. The smaller circle indicates the centroid for the sdM stars, while the larger circle is that for the esdM stars. Stars with a velocity component $V>-220 \mathrm{~km} \mathrm{~s}^{-1}$ are on prograde orbits, while those with $V>-220 \mathrm{~km} \mathrm{~s}^{-1}$ are on retrograde orbits. The tendency for the usdM stars to occupy orbits with large retrograde rotations is clear. Right: Distribution of $W$ vs. $U$ velocities for esdM stars (filled triangles) and usdM stars (open circles) stars. Note the higher energy orbits associated with the usdM stars, suggesting membership in the outer-halo population.

\section{References}

Abadi, M. G., et al. 2003, ApJ, 597, 21

Abazajian, K. N., et al. 2009, ApJS, 182, 543

Adelman-McCarthy, J. K. et al. 2007, ApJS, 172, 634

Allende Prieto, C. et al. 2008, AJ, 136, 2070

An, D., et al. 2009a, ApJ, 700, 523

An, D., et al. 2009b, ApJL, submitted (ArXiv:0907.1082)

Beers, T. C. et al. 1985, AJ, 90, 2089

Beers, T. C. et al. 1992, AJ, 103, 1987

Brook, C. et al. 2007, ApJ, 658, 60

Carollo, D., et al. 2007, Nature, 450, 1020

Carollo, D., et al. 2009, ApJ, submitted (ArXiv:0903.3019)

Christlieb, N., et al. 2008, A\&A A, 484, 721

Ivezić, Ž., et al. 2008a, ApJ, 684, 287

Ivezić, Ž., et al. 2008b, ArXiv:0805.2366

Johnston, K. V., et al. 2008, ApJ, 689, 936

Kazantzidis, S. et al. 2008, ApJ, 688, 254

Lee, Y. S., et al. 2008a, AJ, 136, 2022

Lee, Y. S., et al. 2008b, $A J, 136,2050$

Lepine, S. 2009, AIP Conf. Proc., 1094, p. 545

Newberg, H. J., et al. 2007, ApJ, 668, 221

Schlaufman, K. C., et al. 2009, ApJ, 703, 2177

Roškar, R., et al. 2008, ApJ, 684, L79

Schönrich, R. \& Binney, J. 2009a, MNRAS, 396, 203

Schönrich, R. \& Binney, J. 2009b, MNRAS, in press (ArXiv:0907.1899)

Tumlinson, J. 2007, ApJ, 664, L63

York, D. G., et al. 2000, AJ, 120, 1579

Zwitter, T., et al. 2008, AJ, 136, 421 